\section{SURVEILLANCE DES GLISSEMENTS DE TERRAIN}

Chaque fois qu'il y a risque de rupture d'un ouvrage ou d'un site naturel, la nécessité de pouvoir décider du moment du déclenchement des opérations de sécurité demande l'installation sur le site de dispositifs de surveillance et d'alerte.

Dans ce cas, on s'attache particulièrement à la mesure des déplacements de la masse de terre ou de roche qui créent le risque.

On indique ici, à côté des procédés classique. ment utilisés par les Laboratoires des Ponts et Chaussées (topographie, inclinomètres, tassomètres), des modes d'exploitation et des types de mesure particuliers (inclinomètres, nivelles).

On présente aussi des appareils récents (tassomètres, détecteurs de ruptures, distancemètres) et des dispositifs d'alerte dont I'utilisation semble devoir se développer.

\section{LANDSLIDES SURVEY}

Everytime there is a risk of upsetting of the equilibrium of a civil engineering work or of a natural site, the necessity to decide the beginning of the security operations ask for the setting up of devices to observe and to alert.

In this case one sticks particularly to the mesure of the displacements of the soil or rock mass which creates the risk.

One points out here, nearby the classic procedures used by the Laboratoires des Ponts et Chaussées (surveying, inclinometer, settlement devices) methods of working out (inclinometer) and particular types of mesure (levels).

One presents also apparatuses (settlement devices, shear strip locator, distance meters) and alarm devices whose use seems to have to spread out. 


\section{Surveillance des glissements de terrain}

\section{par B. PINCENT}

Les méthodes classiques de calcul à la rupture fondées sur les résultats de reconnaissances en place et en laboratoire permettent à l'Ingénieur de déterminer avec une précision généralement suffisante l'état de stabilité d'un ouvrage en terre et de dimensionner le cas échéant les systèmes correctifs nécessaires pour assurer ou accroître cette stabilité.

Il est par contre très démuni face au calcul ou à la précision de la chronologie et de la cinématique des mouvements. Chacun ressent bien la nécessité de pouvoir déterminer, chaque fois qu'il y a risque de rupture d'un ouvrage ou d'un site naturel, une frontière critique dans l'espace des paramètres définissant l'équilibre des masses potentiellement instables. Le franchissement de cette frontière serait considéré comme critère d'alerte et déclencherait les opérations de sécurité prévues en conséquence.

La définition précise d'une telle frontière, seuil d'alerte, est encore du domaine du futur. Cela n'empêche pas de tenter de l'approcher empiriquement en développant des méthodes de surveillance dans le triple but de :

- mettre au point des technologies fiables ;

- acquérir des observations expérimentales pour le développement des théories de calcul de déformations ;

- assurer la surveillance des sites critiques même si l'on n'est pas en mesure de définir a priori la conduite à tenir face au phénomène surveillé.

Parmi les paramètres liés directement au comportement de l'ouvrage, déplacements, pressions interstitielles, contraintes par exemple, seuls les déplacements sont directement mesurables.

Les contraintes ou les pressions sont en effet des grandeurs purement théoriques qui ne peuvent être mesurées que par l'introduction d'un appareil qui peut perturber sensiblement la grandeur qu'il est sensé appréhender.

Enfin, ce sont les déplacements de la masse de terre ou de roche qui créent le risque.

Dans cet esprit, les méthodes de surveillance portent essentiellement sur la mesure des déplacements, en surface et en profondeur.

On indique ici les procédés classiquement utilisés par les laboratoires des Ponts et Chaussées, en développant certains modes d'exploitation rationnels des mesures, notamment pour l'inclinomètre. On mentionne aussi quelques types de mesures plus sophistiquées dont l'utilisation semble devoir se développer à l'avenir.

\section{A. LES DEPLACEMENTS DES TERRAINS INSTABLES}

L'évolution des différentes composantes du déplacement de points d'un site instable est très variable dans le temps. On peut cependant remarquer, sans que cela soit une règle absolue, qu'un remblai ou un déblai ou encore une pente naturelle se déforment au cours du temps en suivant trois phases d'évolution (fig. 1):

a) une période de déformation lente où la vitesse des déplacements est pratiquement constante ;

b) une période transitoire où les mouvements s'accélèrent ;

c) la rupture proprement dite où la vitesse du point est grande donnant à la courbe déplacement-temps une ailure asymptotique ;

d) on peut parfois assister à une stabilisation temporaire ou définitive consécutive à un changement dans les causes initiatrices du mouvement, c'est par exemple le cas des pentes naturelles instables dont l'évolution est conditionnée par les cycles météorologiques.

Si l'allure du phénomène est semblable d'un site à l'autre, par contre l'amplitude des déplacements et la durée des différentes périodes peuvent varier dans des proportions considérables ainsi que l'indique le tableau 1 relatif à des ouvrages anciens qui présentent des désordres plus de cinquante ans après leur mise en œuvre.

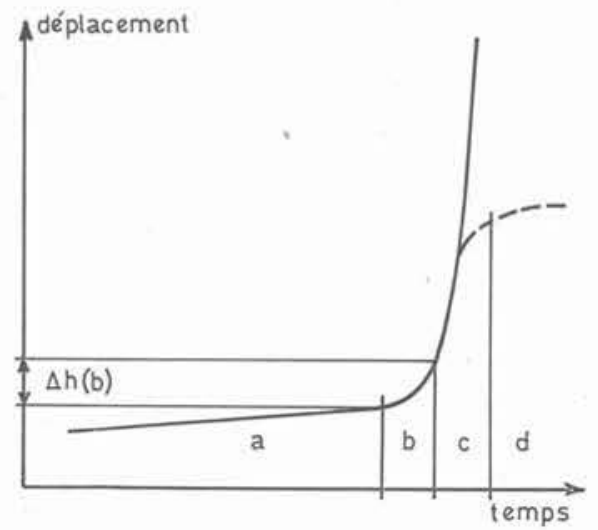

Fig. 1. - Evolution du déplacement d'un point d'un site instable. 
TABLEAU I

Evolution du déplacement vertical de la crête de quatre remblais instables à long terme

\begin{tabular}{|c|c|c|c|c|c|}
\hline Ouvrage & Hauteur & $\begin{array}{l}\text { Matériau } \\
\text { du remblai }\end{array}$ & $\begin{array}{c}\text { Vitesse } \\
\text { en } a\end{array}$ & durée de $b$ & $\Delta h(b)$ \\
\hline Remblai & $8 \mathrm{~m}$ & $\begin{array}{l}\text { sableux } \\
\text { et limono- } \\
\text { argileux }\end{array}$ & $10 \mathrm{~mm} / \mathrm{mois}$ & - & - \\
\hline Remblai & $7 \mathrm{~m}$ & $\begin{array}{l}\text { argileux, } \\
\text { calcaro- } \\
\text { marneux }\end{array}$ & $\begin{array}{l}31 \mathrm{~mm} / \mathrm{mois} \text { * } \\
7 \mathrm{~mm} / \mathrm{mois}\end{array}$ & $\sim 5$ jours & $\sim 30 \mathrm{~mm}$ \\
\hline Remblai & $8 \mathrm{~m}$ & argileux & $2 \mathrm{~mm} / \mathrm{mois}$ & $\sim 20 \quad$ jours & $\sim 50 \mathrm{~mm}$ \\
\hline Remblai & $8 \mathrm{~m}$ & $\begin{array}{l}\text { argileux } \\
\text { calcaro- } \\
\text { marneux }\end{array}$ & $58 \mathrm{~mm} / \mathrm{mois} * *$ & $\sim 0.75$ jour & $\sim 25 \mathrm{~mm}$ \\
\hline
\end{tabular}

(") Point critique.

(**) Stabilisation temporaire avant rupture.

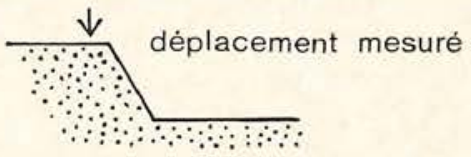

\section{B. MESURE DES DEPLACEMENTS}

\section{A l'intérieur du sol}

Les mouvements de surface d'un site instable sont la traduction de déformations plus profondes qu'il convient d'abord de localiser et ce, le plus rapidement possible, pour le calcul et la définition de solutions confortatives.

Il est quelquefois possible de mesurer de telles déformations directement sur un ouvrage du site à l'aide d'un puits par exemple (fig. 2), mais le plus souvent, il est nécessaire d'installer un capteur dans le sol. Pour cela, on emploie :
- les tassomètres pour la mesure des tassements verticaux ;

- les extensomètres pour celle des déformations ;

- les inclinomètres pour celle des déplacements horizontaux du sol.

\section{Le tassomètre}

Il mesure le déplacement vertical d'un point dans le sol. Le principe de l'appareil repose :
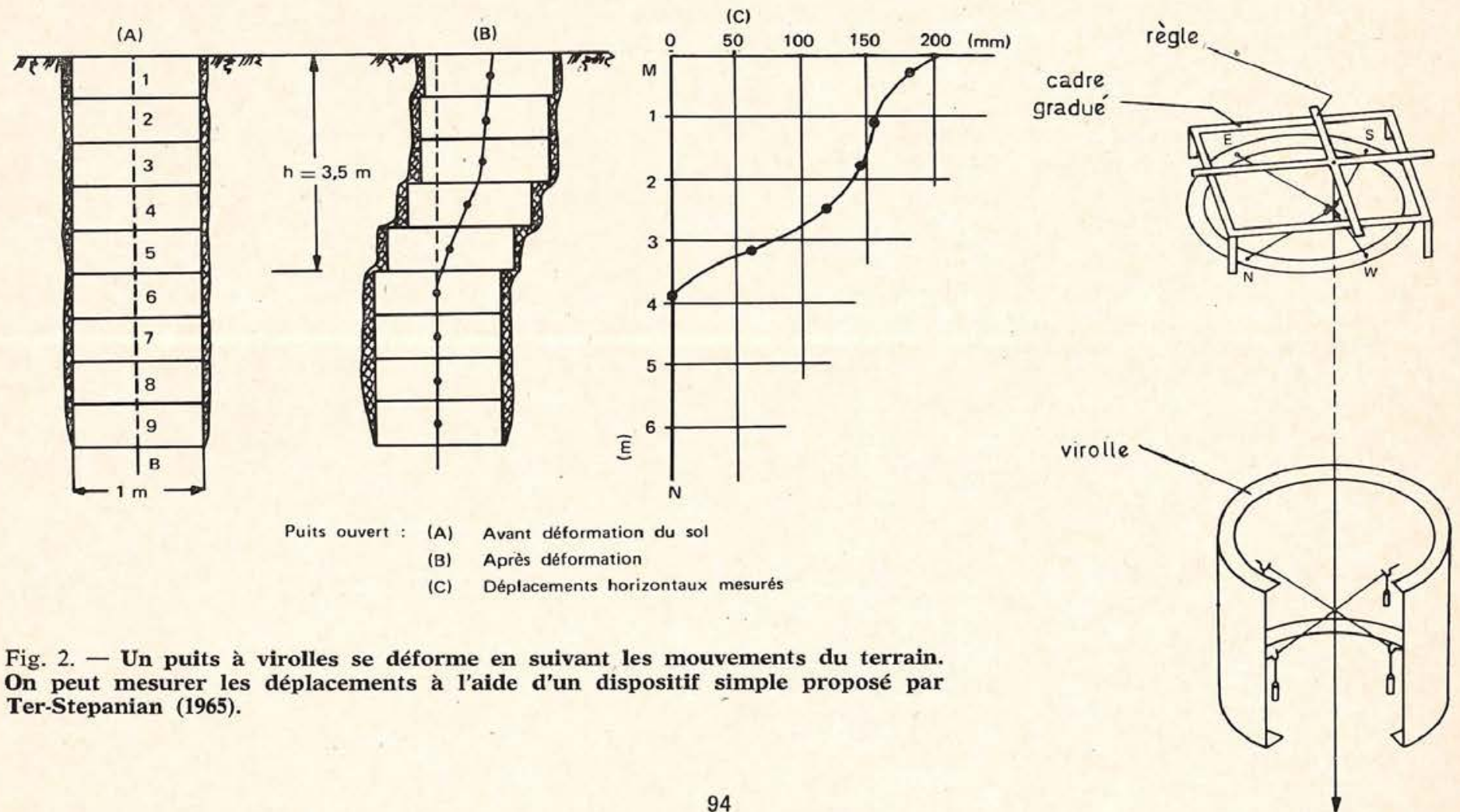

Fig. 2. - Un puits à virolles se déforme en suivant les mouvements du terrain. On peut mesurer les déplacements à l'aide d'un dispositif simple proposé par Ter-Stepanian (1965). 


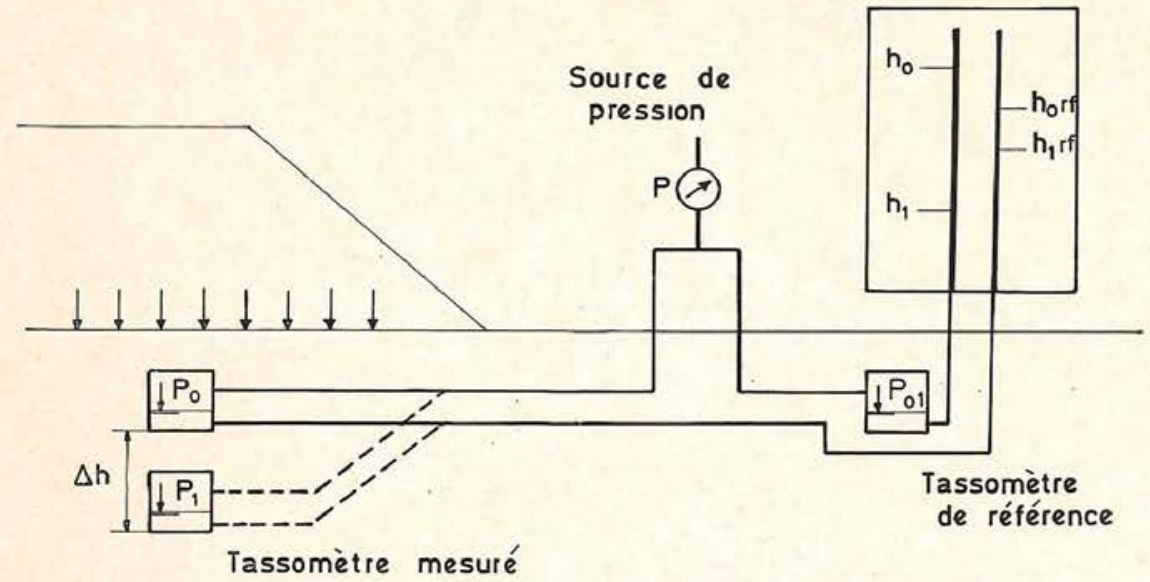

Fig. 3. - Schéma d'installation d'un tassomètre.

soit sur la mesure de la pression d'une colonne de liquide dont une extrémité est placée au point qui tasse et l'autre en un point de cote connue si possible fixe (fig. 3) ;

- soit sur la détermination de la position de bagues (magnétiques ou non) solidaires du sol à l'extérieur d'un tube de sondage ou encore de balles radioactives tirées dans le sol à partir d'un canon descendu dans un tube de forage puis localisées par diagraphie (fig. 4 - Procédé Syminex).

L'erreur de mesure est de $\pm 2 \mathrm{~mm}$ pour ces deux procédés.

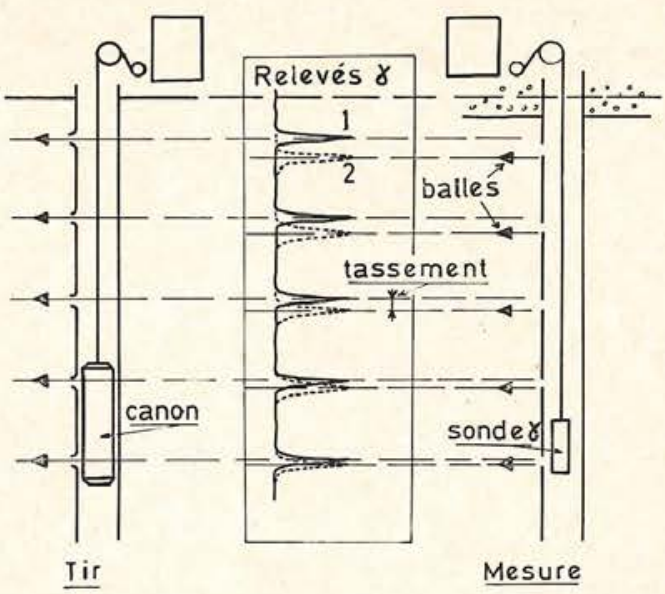

Fig. 4. - Tassomètre à balles radioactives (procédé Syminex, dessin d'après Technical Doc. 201. SyminexJanuary 1976).

mesure de l'angle d'inclinaison de la torpille, en des points régulièrement espacés d'une longueur $a$, on peut reconstituer, par sommation, la déformée du tube au moment de la mesure. Ceci, à condition de connaître simultanément la position d'un point du tube :

- le pied si on peut le supposer fixe ;

- la tête si on la suit par topographie.

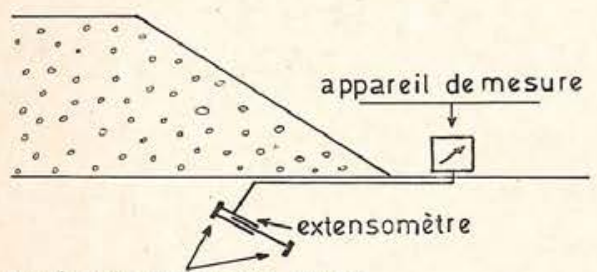

extrémités solidaires du sol

Fig. 5. - Principe d'utilisation de l'extensomètre.

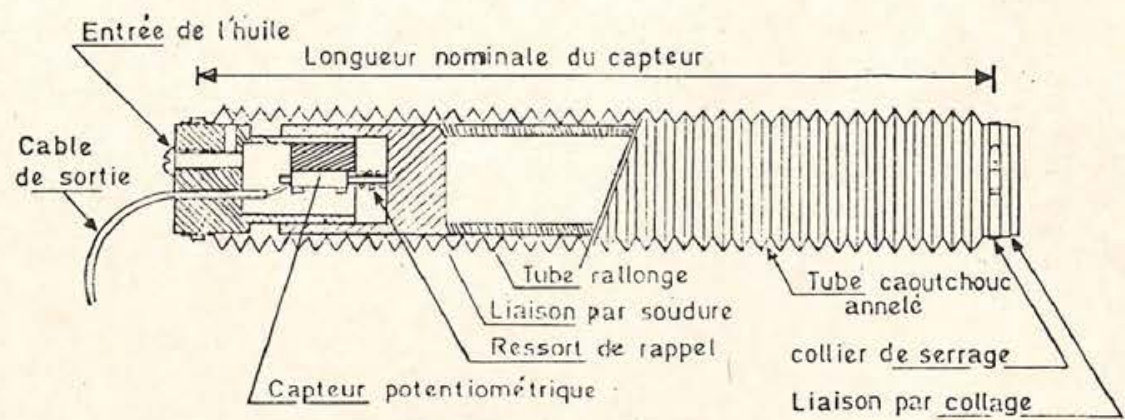

Fig. 6. - Schéma d'un extensomètre.

\section{L'extensomètre}

Cet appareil mis au point par le laboratoire régional de Bordeaux et utilisé sous le remblai expérimental à la rupture de Cubzac-les-Ponts mesure le déplacement relatif de deux points dans le sol (fig. 5 et 6).

C'est un tube télescopique dont les déformations sont mesurées par potentiomètre. L'erreur sur le déplacement relatif est de $\pm 1 / 10$ de $\mathrm{mm}$ après étalonnage préalable du potentiomètre. La longueur du capteur est, selon le type, de $0.5 \mathrm{~m}$ ou $1 \mathrm{~m}$.

\section{L'inclinomètre}

L'inclinomètre (fig. 7) est un appareil bien connu dont l'objet est de déterminer la déformée au cours du temps d'un tube placé verticalement dans le sol et, si possible, ancré dans le substratum sain.

Une « torpille » coulissant juste dans le tube est descendue à l'intérieur de celui-ci et donne son inclinaison par rapport à la verticale. A partir de la

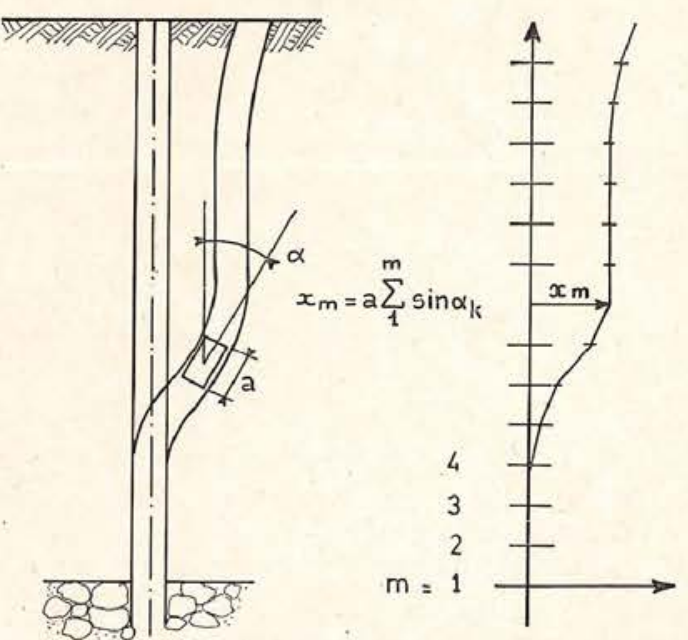

Fig. 7. - Principe de l'inclinomètre. 
La répétition de ces mesures au cours du temps donne l'évolution des déplacements horizontaux dans le sol (fig. 8).

Cependant, si le principe est simple, l'exploitation des mesures est parfois délicate.

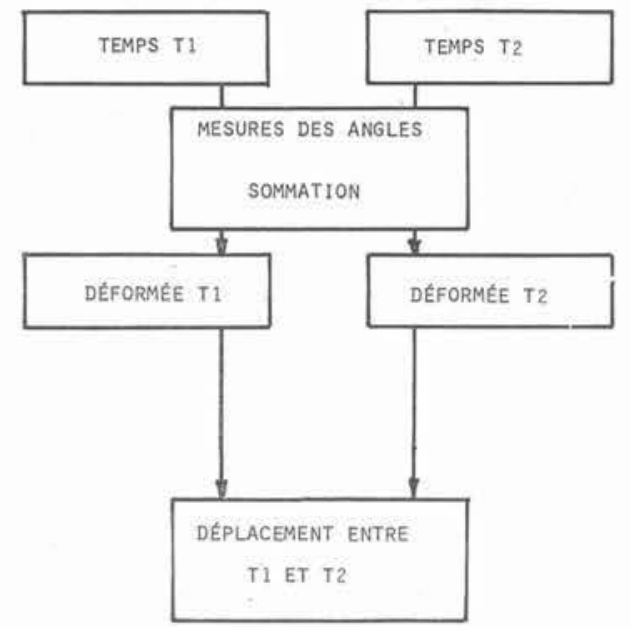

Fig. 8. - Reconstitution de la déformée et des déplacements à partir de la mesure des angles.

Un calcul élémentaire montre que si la mesure des angles $x$, à l'instant $t$, est entachée d'une erreur $\varepsilon(t)$, constante au cours de la mesure, le déplacement calculé au point $m$ entre les instants $t_{1}$ et $t_{2}$ est entaché de l'erreur $m\left(\varepsilon\left(t_{1}\right)+\varepsilon\left(t_{2}\right)\right)$.

Prenons, par exemple, le cas d'un tube placé parfaitement vertical au temps $t_{1}$ et le restant jusqu'au temps $t_{2}$.

Les mesures à $t_{1}$ et $t_{2}$ donneraient une valeur d'angle située dans une bande d'erreur de largeur $\pm \varepsilon(t)$ tandis que les déformées obtenues après sommation des mesures se trouveront quelque part dans le triangle $\mathrm{ABC}$ de base $\mathrm{AB}=2 m \varepsilon(t)$, le point $\mathrm{C}$ étant supposé fixe (fig. 9).

Fig. 9. - Valeur vraie et valeur mesurée pour les angles $\alpha$ et, pour la déformée, dans un tube vertical.
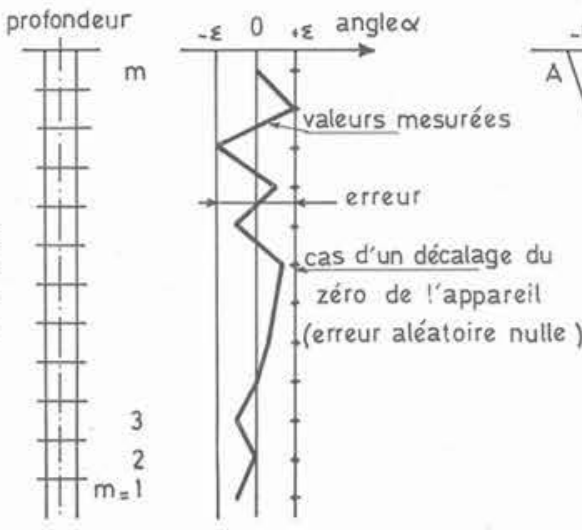

Les déplacements horizontaux, différence entre deux déformées de ce type, se placeront dans un triangle de base $2 m\left(\varepsilon\left(t_{1}\right)+\varepsilon\left(t_{2}\right)\right)$.

Le décalage de zéro de l'appareil (effet de la dérive par exemple) est très courant et donne lieu à ce type d'erreur. Il fait croire alors à une déformation d'ensemble du terrain qui en fait n'a pas lieu (fig. 9).

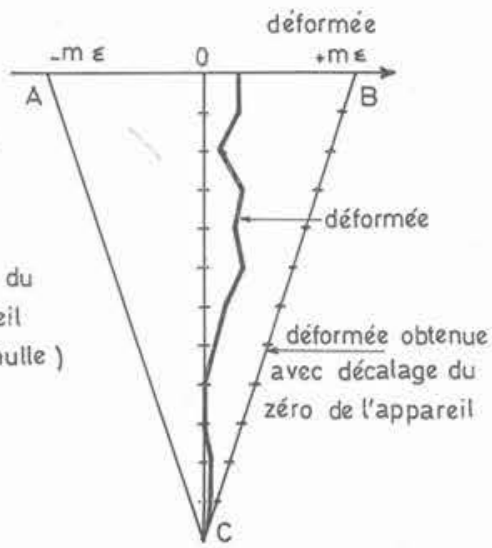

\subsection{Diminution de l'erreur $\varepsilon$}

L'erreur $\varepsilon$ est la somme de l'erreur aléatoire $\varepsilon_{a}$ et de l'erreur systématique $\varepsilon_{1}$.

La valeur réelle de l'angle s'exprime par :

$$
x \text { réel }=x \text { mesuré }-\varepsilon_{a}-\varepsilon_{s}
$$

On peut supprimer l'erreur systématique, due le plus souvent à la dérive des appareils, en effectuant pour chaque direction principale du tube inclinométrique deux mesures " à $180^{\circ}$ " (fig. 10).

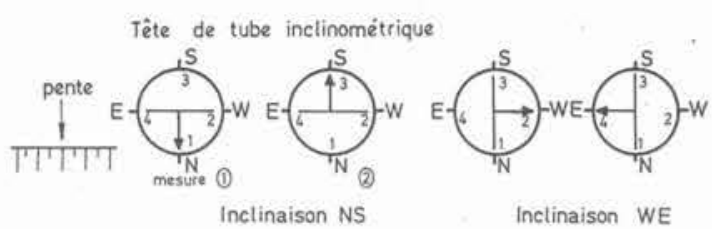

Fig. 10. - Le relevê des faces 1-3 et $\mathbf{4 - 2}$ permet de supprimer l'erreur systématique par le calcul.

L'angle réel change alors de signe, l'erreur systématique reste identique. On a les deux égalités :

$x$ réel à $0^{\circ}=x$ mesuré $\left(0^{\circ}\right)-\varepsilon_{a}\left(0^{\circ}\right)-\varepsilon_{s}$

$x$ réel $180^{\circ}=-x$ réel $0^{\circ}=$

$$
=x \text { mesuré }\left(180^{\circ}\right)-\varepsilon_{a}\left(180^{\circ}\right)-\varepsilon_{s}
$$

En supposant que $\varepsilon_{a}\left(0^{\circ}\right)=\varepsilon_{a}\left(180^{\circ}\right)=\varepsilon_{a}$ et que $\varepsilon_{\text {s }}$ est constant pendant la mesure, la somme et la différence de (1) et (2) donnent :

$2\left(\varepsilon_{s}+\varepsilon_{a}\right)=x$ mesuré $\left(0^{\circ}\right)+x$ mesuré $\left(180^{\circ}\right)$

$2\left(x\right.$ réel $\left.+\varepsilon_{a}\right)=x$ mesuré $\left(0^{\circ}\right)-x$ mesuré $\left(180^{\circ}\right)$

On obtient donc par cette méthode :

a) la valeur de l'erreur systématique entachée de l'erreur aléatoire seule. Un calcul statistique sur la quantité $\left(\varepsilon_{s}+\varepsilon_{a}\right)$ pour un relevé $\left(0^{\circ}\right.$ et $\left.180^{\circ}\right)$ fournit la moyenne de $\varepsilon_{s}$ et l'écart type $\sigma$ de la population :

b) la valeur de l'angle $\alpha$ entachée de l'erreur aléatoire seule.

Cette méthode, mise au point au laboratoire régional de Toulouse et testée sur des cas réels à l'aide d'un inclinomètre Soils Instruments, se concrétise par la mise au point d'un programme de dépouillement donnant pour la mesure des angles $\alpha$ :

- la valeur de l'angle $\alpha$ au point de mesure;

- l'erreur systématique prise égale à la moyenne des quantités $\varepsilon_{a}+\varepsilon_{s}$ 
- l'erreur aléatoire égale en module à $2 \sigma$, $\sigma$ étant l'écart type de la population $\varepsilon_{a}+\varepsilon_{s}\left(2 \sigma \simeq 0.016^{\circ}\right.$ pour un tube de $34 \mathrm{~m}$ de long). Ce dernier est un excellent "indice de qualité » de la mesure et de l'appareillage.

De ce calcul appliqué à différents relevés dans le temps, on déduit :

- le déplacement angulaire à un instant $t$ par rapport à un relevé origine et son erreur ;

- le déplacement horizontal différence entre la déformée à l'instant $\mathrm{t}$ de la déformée origine, et son erreur.

\subsection{Intérêt de l'interprétation de la courbe des angles $\alpha$}

La définition de la courbe $\alpha$ fonction de la profondeur est plus précise que celle de la déformée.

Exploiter la courbe $\alpha$ (profondeur) plutôt que la déformée présente un autre avantage : la détermination précise des profondeurs de rupture.

En effet, des ruptures idéales, telles que celles représentées figure 11 donnent une courbe des angles $\alpha$ qui présente des pics dont les sommets correspondent aux lignes de rupture tandis que la déformée indique moins clairement la position exacte de la ligne de rupture.

Dans le cas de mesures réelles où les courbes sont entachées de «bruit», la distinction est encore plus évidente (fig. 12).

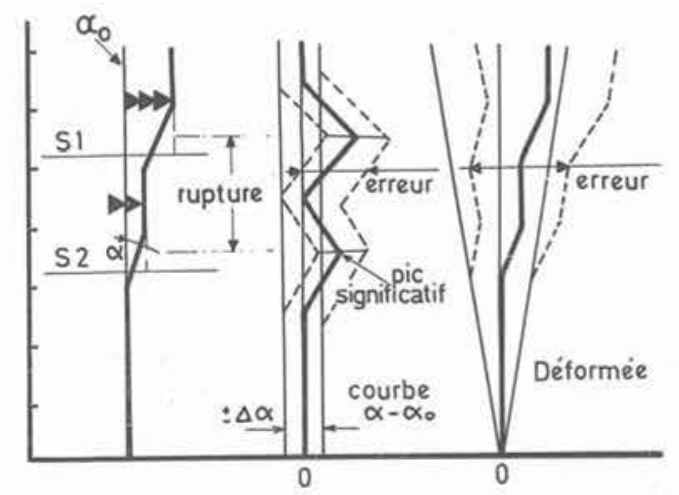

Fig. 11. - Interprétation des mesures inclinométriques avec introduction de l'erreur de mesure : la position de la surface de rupture est plus explicite sur la courbe des $\alpha$ que sur la déformée.

\subsection{Evolution des courbes en $\alpha$ avec le temps}

Les courbes de déplacement angulaire $\alpha-\alpha_{o}$ (où $\alpha$ est l'angle mesuré à l'instant $t$ et $\alpha_{0}$ l'angle mesuré à l'instant initial) représentées figure 12 sont tirées des mesures effectuées sur un inclinomètre placé en tête d'un remblai de $40 \mathrm{~m}$ de haut, depuis longtemps le siège de mouvements plus ou moins importants. Elles mettent en évidence une rupture à $23 \mathrm{~m}$ de profondeur, dans une couche d'argile, et d'autres phénomènes qui font apparaître des pics sur les courbes $\alpha-\alpha_{0}$ et qui sont provoqués par :

- une lecture erronée ou une mauvaise position de la sonde dans le tube : ils sont décelables par la valeur anormale de l'erreur $\varepsilon_{a}+\varepsilon_{s}$ calculée en ce point ;

- la mise en place progressive du tube dans le forage ou par le flambement du tube sous son propre poids. Dans ce cas, l'évolution des courbes présente un caractère "désordonné " qui permet de les distinguer des pics qui représentent une rupture réelle.

\section{Déplacements de la surface du sol}

Les mouvements de la surface du sol sont les plus aisément accessibles. L'expérience montre cependant que la précision obtenue dans ces mesures est loin de celle attendue par simple déduction des performances de l'appareil de mesure (théodolite, chambres de photogrammétrie, capteurs de déplacement, etc.). La perte de précision se fait au niveau de chaque maillon de la chaîne de mesure et particulièrement à l'amont de la mesure elle-même (conception, mise en place des repères, des bases de référence...) et non au niveau des appareils.

\section{Mesures directes}

Les mesures manuelles directes de distance entre des repères rapprochés sont simples, économiques et précises : une règle graduée ou un pied à coulisse assurent une erreur relative de l'ordre de $10^{-3}$. Les distances mesurées sont des distances relatives, limitées à quelques dizaines de centimètres et à des sites aisément accessibles.

Pour la mesure précise du déplacement de deux points éloignés d'une centaine de mètres et lorsque la configuration du terrain s'y prête, on peut envisager d'utiliser un fil ou ruban tendu suivant la figure 13 .

\section{Mesure de distance par voie optique}

La topométrie de site instable consiste à suivre le déplacement dans le temps de points matérialisés par des repères. Pour cela, on dispose de différentes méthodes :

- topographie traditionnelle à l'aide d'un théodolite ;

- photogrammétrie ;

- mesure de distances avec un distancemètre électrooptique.

La mesure des angles à l'aide d'un théodolite (fig. 14) permet de déceler des mouvements de $0,16 \mathrm{~mm}$ à $100 \mathrm{~m}\left(10^{-4}\right.$ grade $)$.

Les expériences de topographie menées par les laboratoires sur des ouvrages (remblais, déblais, pentes naturelles instables) n'ont jamais permis d'obtenir une erreur inférieure à $\pm 5 \mathrm{~mm}$ sur des déplacements à une cinquantaine de mètres (déblai de Bosse Calin, remblai expérimental de Cubzac-les-Ponts). Elles étaient faites, il faut le préciser, à partir de deux stations seulement, sans dispositif de centrage forcé, chaque station visant simultanément un point matérialisé par un clou sur un piquet de bois. L'erreur est dans la majorité des cas courants de l'ordre de $\pm 2 \mathrm{~cm}$.

Les causes d'erreur sont diverses et diffuses. Elles ne mettent en cause ni le principe, ni les appareils utilisés, mais plutôt l'«implantation » de la topographie en site instable qui pose des problèmes spécifiques. On peut citer comme cause d'erreurs pouvant limiter la qualité des mesures:

- l'instabilité du terrain où sont placées les stations ;

- l'influence des mouvements du sol superficiel (variation d'humidité, de température) sur une borne insuffisamment ancrée en profondeur (fig. 15) ;

- le retrait du béton d'une borne utilisée trop tôt ;

- le positionnement inexact du théodolite sur la station : un dispositif de centrage forcé installé à demeure sur les piliers de base évite les erreurs de position à chaque remise en station ; 


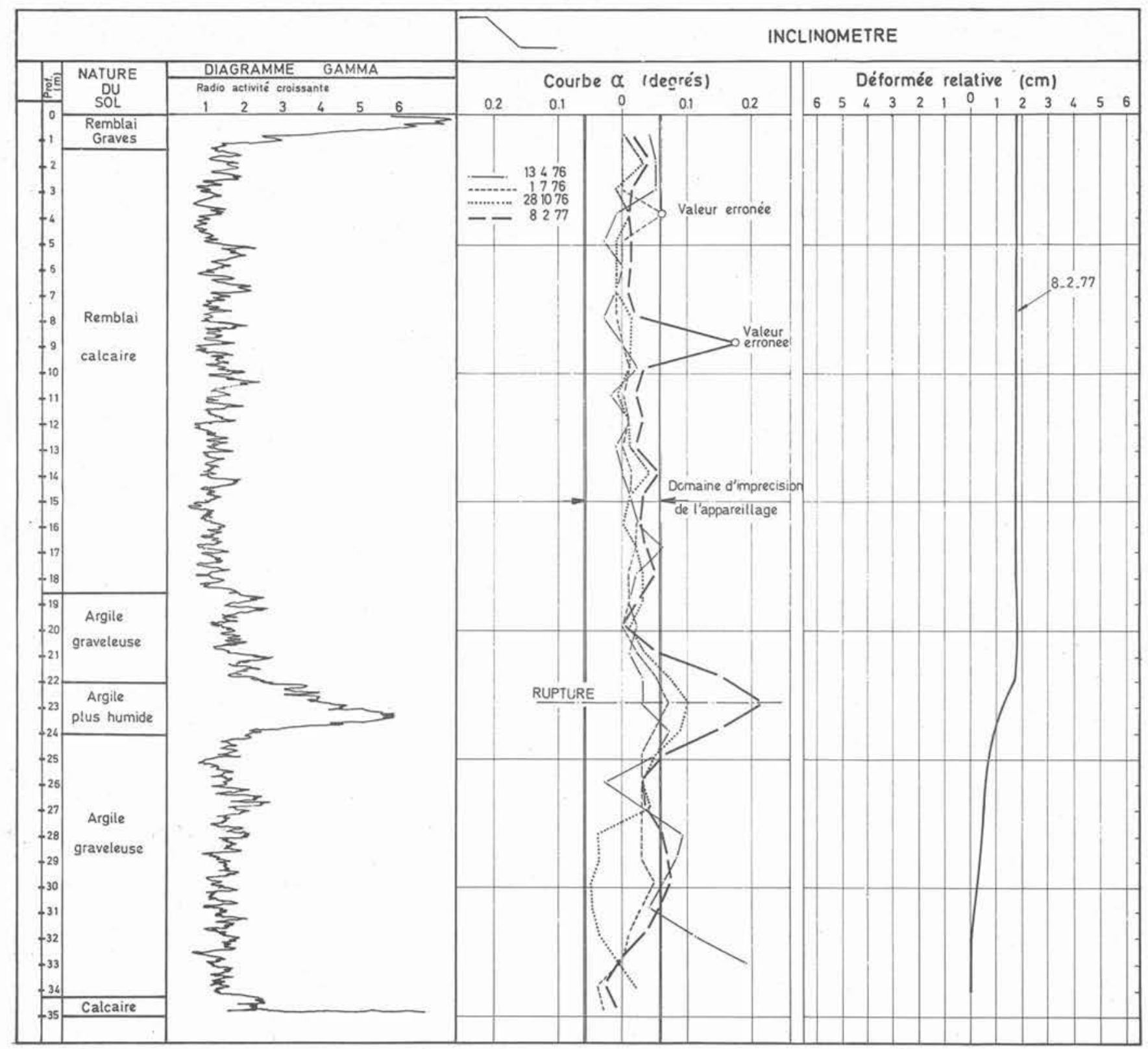

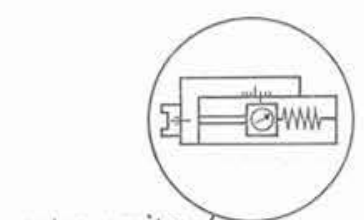

extensomètre

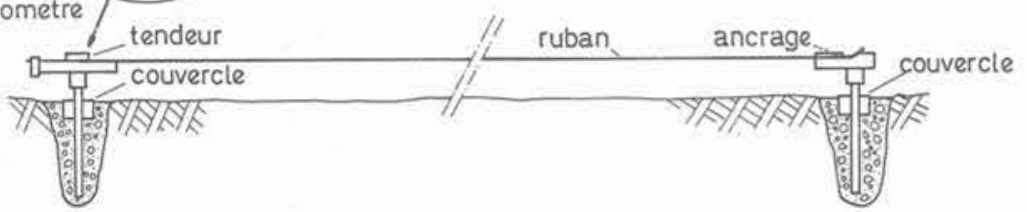

Fig. 13. - Mesure précise du déplacement de deux points. L'appareil situé à l'extrémité gauche du ruban permet d'appliquer une tension constante au ruban et de mesurer son déplacement.
Fig. 12. - Dépouillement de mesures inclinométriques. On peut noter la position de la rupture mise en évidence par les courbes $\alpha=\alpha_{0}$, dans une couche très argileuse (pic de rayonnement $\gamma$ ).

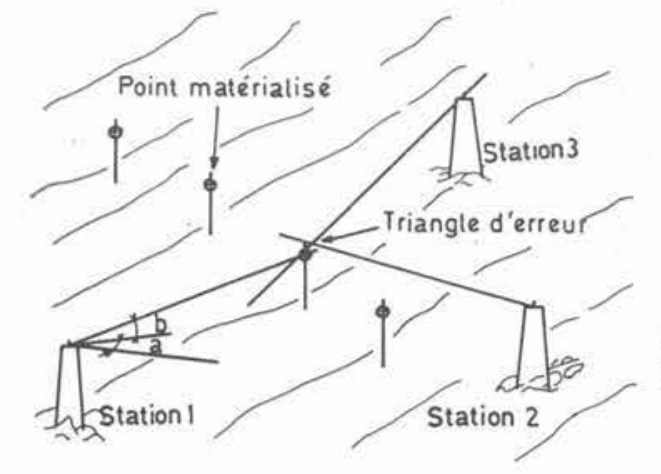

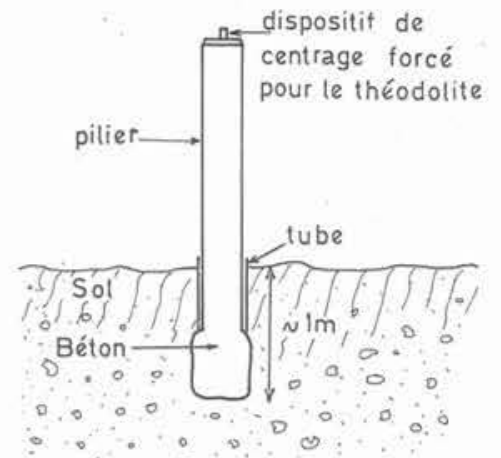

Fig. 15. - Schéma d'un pilier de station insensible aux mouvements superficiels du terrain.

Fig. 14. - Topographie à l'aide d'un théodolite (principe). 
- un nombre de stations insuffisant : deux sont indispensables, trois ou quatre permettent de contrôler la qualité de la mesure ;

- la non-simultanéité des mesures des angles d'un point se déplaçant entre la mesure du premier angle et celle du dernier ;

- la qualité des cibles placées sur les repères : leur dessin doit éviter toute ambiguïté de pointe même à longue distance ;

- le changement d'équipe ou de matériel ou, pire, de méthode entre deux relevés.

\section{La photogrammétrie}

La photogrammétrie est l'ensemble des techniques qui permettent de définir la forme, les dimensions et la position d'un objet à partir de perspectives de cet objet enregistrées photographiquement.

L'exploitation des clichés s'appelle restitution photogrammétrique. Elle n'est possible que si la position spatiale d'un canevas de base constitué d'au moins trois points du cliché est connue sur le terrain (fig. 16 et 17).

Il existe deux types de restitution :

a) La restitution analogique.

Elle se fait sur un restituteur permettant le pointé stéréoscopique et qui donne directement la position spatiale du point. Cette stéréophotogrammétrie impose des conditions restrictives à la prise de vues : des échelles sensiblement égales pour les deux

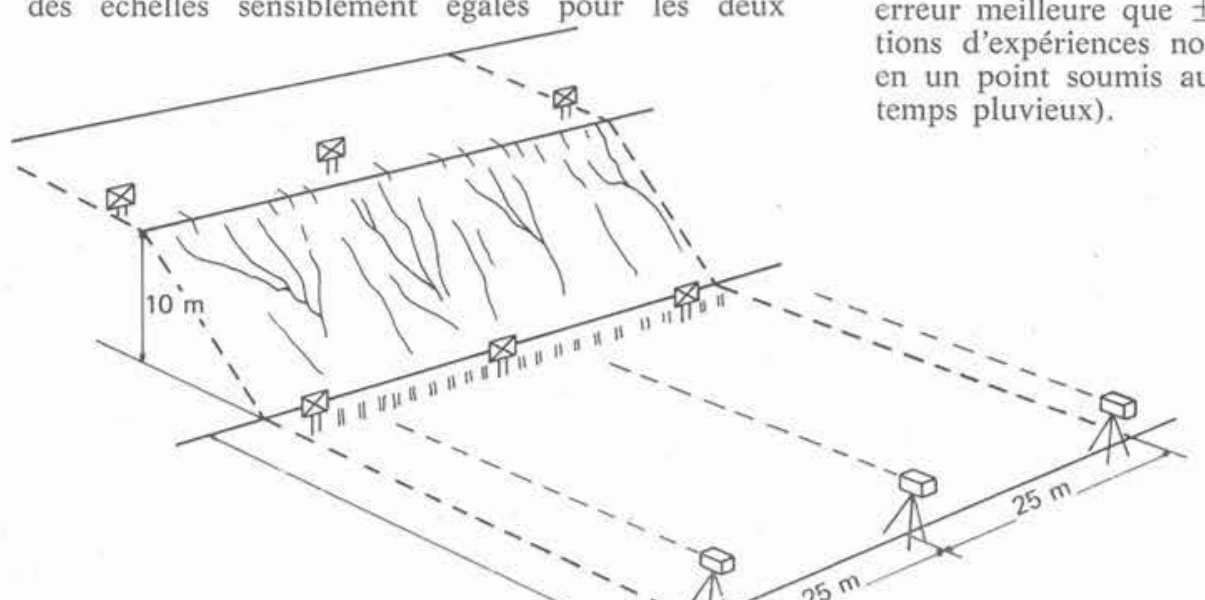

Fig. 16. - Photogrammétrie terrestre. Schéma de prise de vues et canevas d'appui. glissement de terrain affectant un talus de déblai de l'autoroute A 51 Aix-en-Provence-Marseille.

Différentes expériences y ont été menées, tant en topographie qu'en photogrammétrie, avec restitution analogique classique et en fausse stéréoscopie (exploitation des clichés pris d'une même base à des instants différents).

Les différents résultats, obtenus principalement au cours de séances où les cibles ont été déplacées de quantités connues entre deux relevés effectués dans la même journée, indiquent des dispersions importantes, sans que les causes aient été clairement mises en évidence jusqu'ici.

\section{Distancemètre électro-optique}

On peut mesurer la distance séparant deux points matériels en émettant du point de base un rayon lumineux modulé (visible ou invisible), réfléchi vers l'émetteur par un miroir (triple prisme) placé au point visé. La distance parcourue par le rayon lumineux est déduite de la différence de phase existant entre la lumière modulée émise et celle du rayon réfléchi.

Des mesures effectuées dans le cadre d'une démonstration dans une configuration "surveillance de talus " d'un appareil très performant de ce type (Mekometer Kern, portée maximale $3 \mathrm{~km}$, erreur sur la distance $\pm\left(0.3 \mathrm{~mm}+2 \cdot 10^{-6} \mathrm{D}\right)$, durée d'une mesure $\left.\simeq 3 \mathrm{mn}\right)$ indiquent qu'il est possible d'obtenir la valeur du déplacement d'un point situé à 260 mètres avec une erreur meilleure que $\pm 0.5 \mathrm{~mm}$. Ceci dans des conditions d'expériences non idéales (appareil sur trépied, en un point soumis au mouvements de la circulation, temps pluvieux). photos et des axes optiques des chambres de prises de vue à peu près parallèles (divergence ou convergence $<10^{\circ}$ ) et impose donc l'utilisation de bases fixes.

b) La restitution analytique.

On mesure les coordonnées planes sur les clichés (IGN $\varnothing$ erreur $3 \mu$ ). La restitution de la position des points est réalisée par le calcul sur ordinateur à partir du canevas de base pris sur les clichés. On s'affranchit par cette méthode des sujétions de positionnement des chambres, ce qui autorise l'utilisation de perspectives intéressantes qui, associées à la puissance des programmes de calcul, donne de meilleurs résultats par cette méthode que par la précédente.

4. Expérimentations de méthodes topographiques et photogrammétriques sur un talus instable.

Une expérimentation de ces méthodes a été réalisée par le laboratoire du CETE d'Aix-en-Provence, sur un

Fig. 17. - Schéma général d'un canevas de prises de vues photogrammétriques.

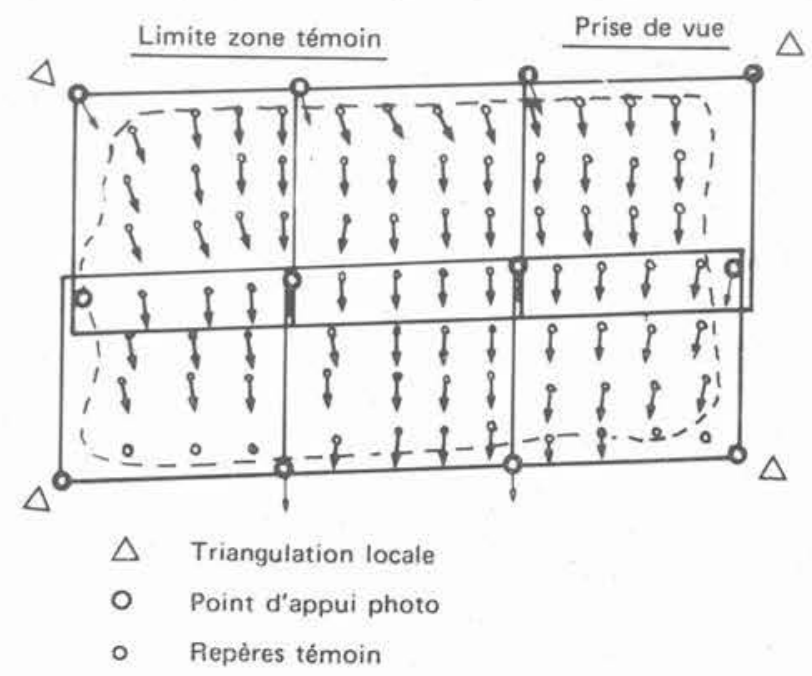




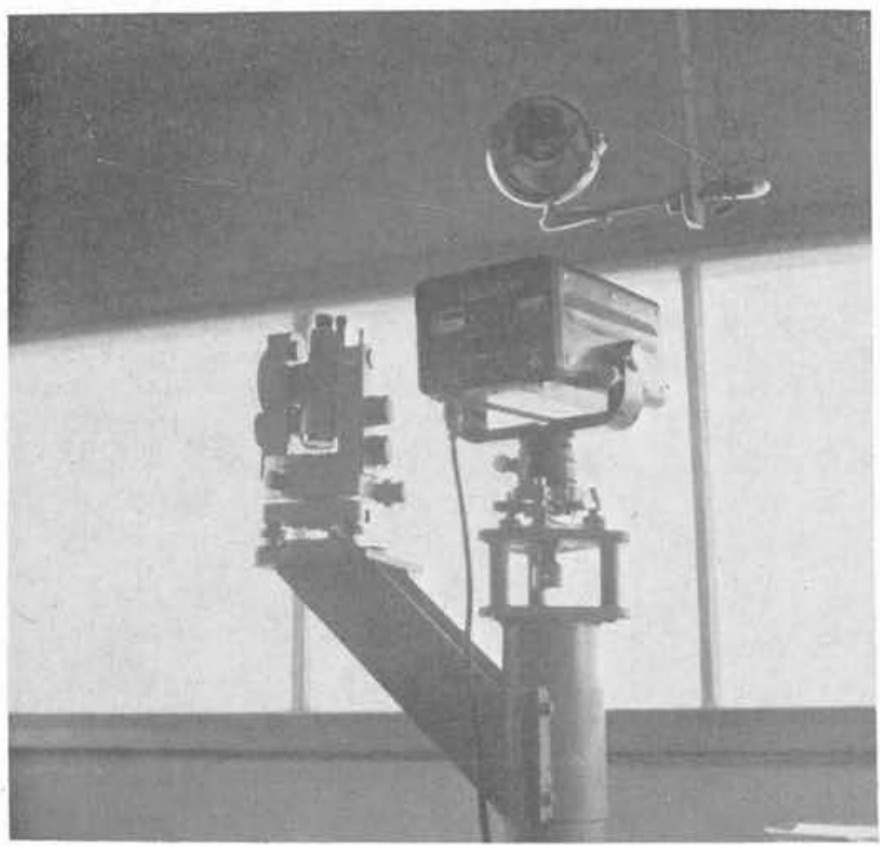

Fig. 18 a, - Le distancemètre utilisé à Asbestos.

6. Un exemple d'utilisation de distancemètre :

Surveillance de la mine à ciel ouvert d'Asbestos (Canada)

La mine d'amiante d'Asbestos (profondeur $250 \mathrm{~m}$, diamètre en tête d'environ $1000 \mathrm{~m}$ ) dispose d'un système de surveillance du mouvement des talus par distancemètre-laser. Celui-ci est associé à un théodolite pointant les cibles en utilisant le rayon laser réfléchi. Le nombre de réflecteurs installés est d'environ soixante. Ils sont placés en moyenne à $700 \mathrm{~m}$ de l'émetteur et ont été observés vingt-quatre heures sur vingtquatre durant certaines périodes critiques à raison de cinquante pointés en trois heures. Le distancemètre placé sur un pilier dans un local climatisé, en crête de talus, a donné des mesures avec une erreur expérimentale de $\pm 6 \mathrm{~mm}$ sur points fixes par temps normal et de $\pm 15 \mathrm{~mm}$ par temps de brume (fig 18 a et b).

La mesure continue était assurée par un roulement de trois appareils, annulant ainsi les effets des pannes et des temps de réparation!

En conclusion, il est possible de mesurer des déplacements par voie optique à l'aide d'appareils divers, très récents comme les télémètres électro-optiques, ou beaucoup plus courants tel le théodolite.

Chacun de ces moyens utilise la lumière comme transport de l'information. Il est donc soumis à certaines contraintes de propagation de la lumière (météorologie, nuit),

Enfin, ces moyens possèdent des caractéristiques, qualités et défauts (fiabilité, temps de mesure, temps d'exploitation, contraintes particulières) qui nécessitent une adaptation réfléchie au problème posé.

\section{Les détecteurs de mouvements}

Les détecteurs de mouvements ne donnent qu'une information partielle. Ils peuvent indiquer l'existence d'un mouvement :

- à l'intérieur du sol : c'est le cas du détecteur de profondeur de rupture et de l'inclinomètre à piges

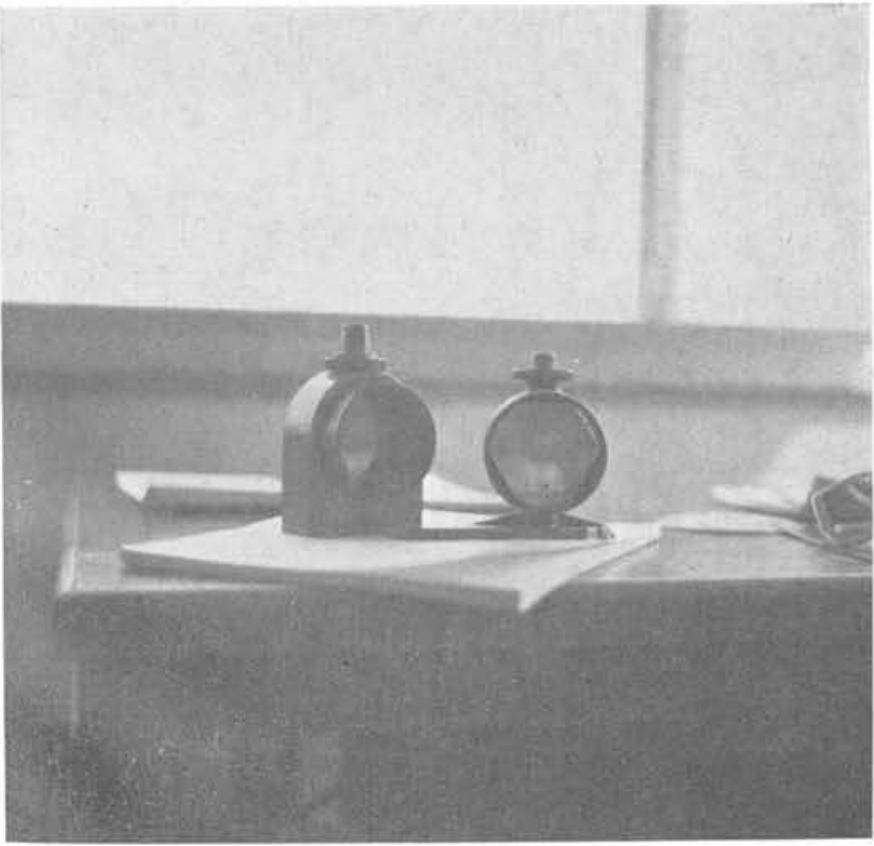

Fig. 18 b. - Les réflecteurs.

qui ne donnent que la profondeur d'une rupture lorsqu'elle se produit ;

- à la surface : c'est le cas des nivelles qui mesurent la rotation d'un point de la surface du terrain et, de ce fait, peuvent donner une indication sur l'accélération des mouvements lorsqu'elle se produit.

\section{Le détecteur de profondeur de rupture}

Cet appareil (fig. 19) très simple dans son principe donne la profondeur d'un mouvement par rupture d'un fil conducteur sur lequel sont fixées, à différentes profondeurs, des résistances électriques. Deux câbles incassables assurent les liaisons électriques en profondeur.

L'ensemble est placé dans un sondage de petit diamètre et tenu en place par un coulis isolant et cassant. La mesure de la résistance entre les fils en tête de sondage donne directement ou par calcul la profondeur des ruptures (Brevet Terrametrics USA).
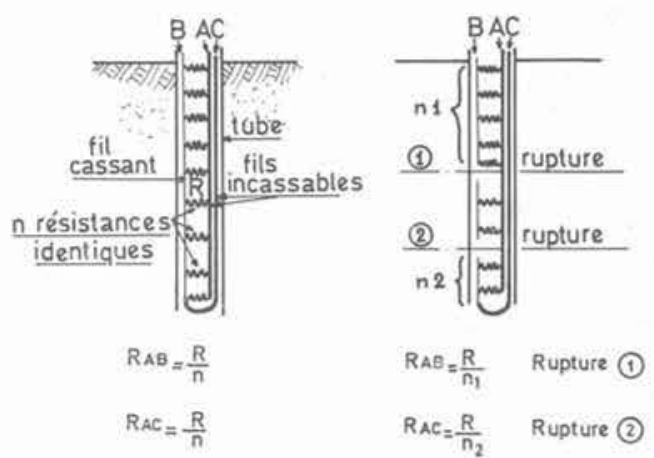

Fig. 19. - Principe du détecteur de profondeur de rupture. Il permet de connaitre la profondeur de zones de rupture.

\section{L'inclinomètre à piges.}

La profondeur d'une surface de glissement est détectée en faisant passer dans un tube inclinométrique lisse des torpilles de diamètre croissant. Une déformation de tube provoquée par un mouvement du sol coince une des piges et indique la zone de rupture. 


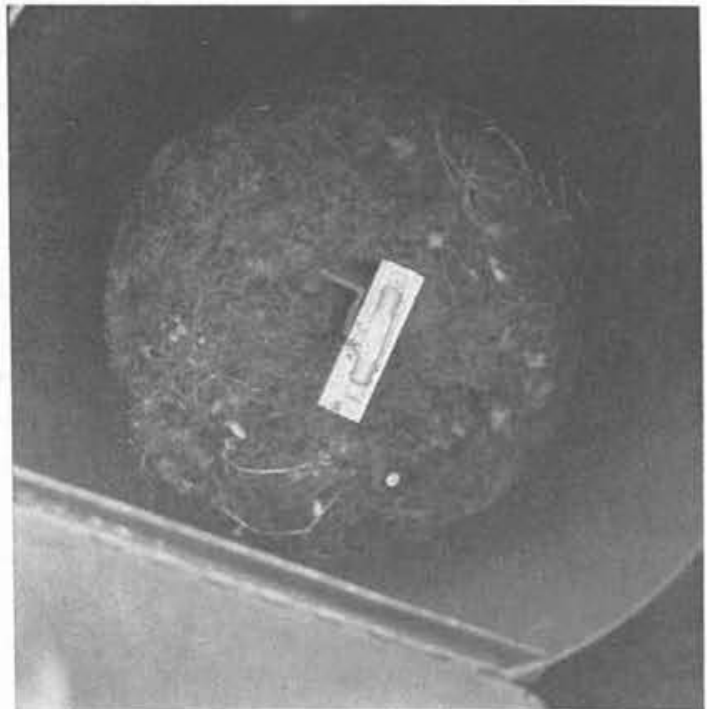

Fig. 20. - Nivelle sur son support (doc. MRN, Québec).

La grande simplicité de cet appareil est compensée par le peu d'informations qu'il fournit et les difficultés de mise en place du tube dans le sondage qui doit être droit au départ.

L'expérience indique que les mouvements ne sont détectables que lorsque leur amplitude est « suffisamment grande " pour "bien déformer " le tube. Dans ce cas, la torpille se coince au premier coude du tube, masquant ainsi les rupture plus profondes. Une solution à ce problème adoptée par le service géotechnique du Ministère des Richesses Naturelles du Québec, consiste à laisser une pige au fond du forage afin de détecter la surface de rupture la plus profonde, qui est généralement la plus intéressante.

\section{Les nivelles}

Ces appareils sont des niveaux à bulle de grande sensibilité placés sur un support lié au sol (fig. 20 et 21).

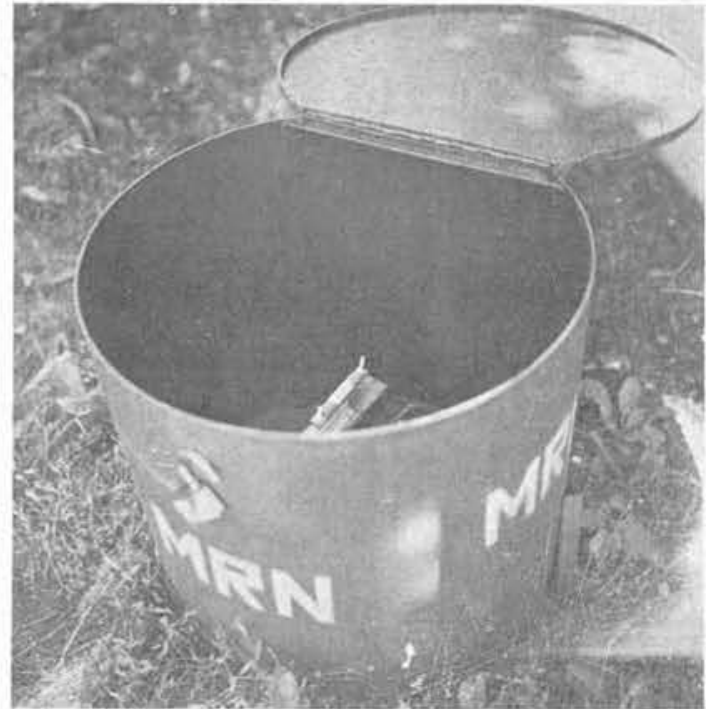

Fig. 21. - Nivelle et support dans leur boitier de protection.

Ils détectent les mouvements du terrain par la mesure de la rotation du point et son évolution au cours du temps.

Le déplacement de la bulle peut être régulièrement relevé par un personnel non spécialisé ; si l'amplitude des mouvements fait craindre un « débullage » (bulle coincée à une extrémité du niveau), l'appareil est relevé puis remis à zéro à l'aide d'un système à vis.

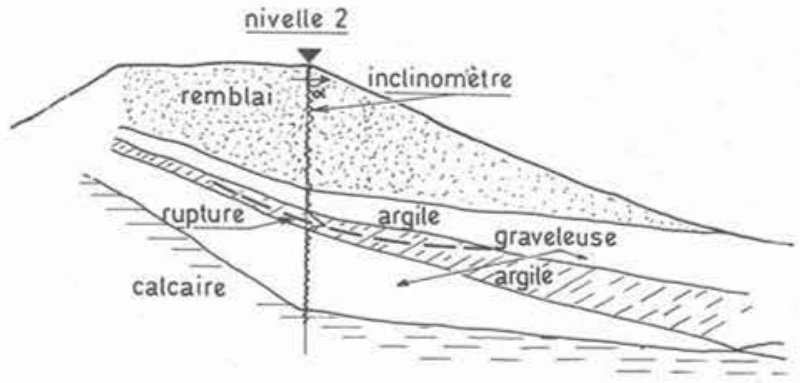

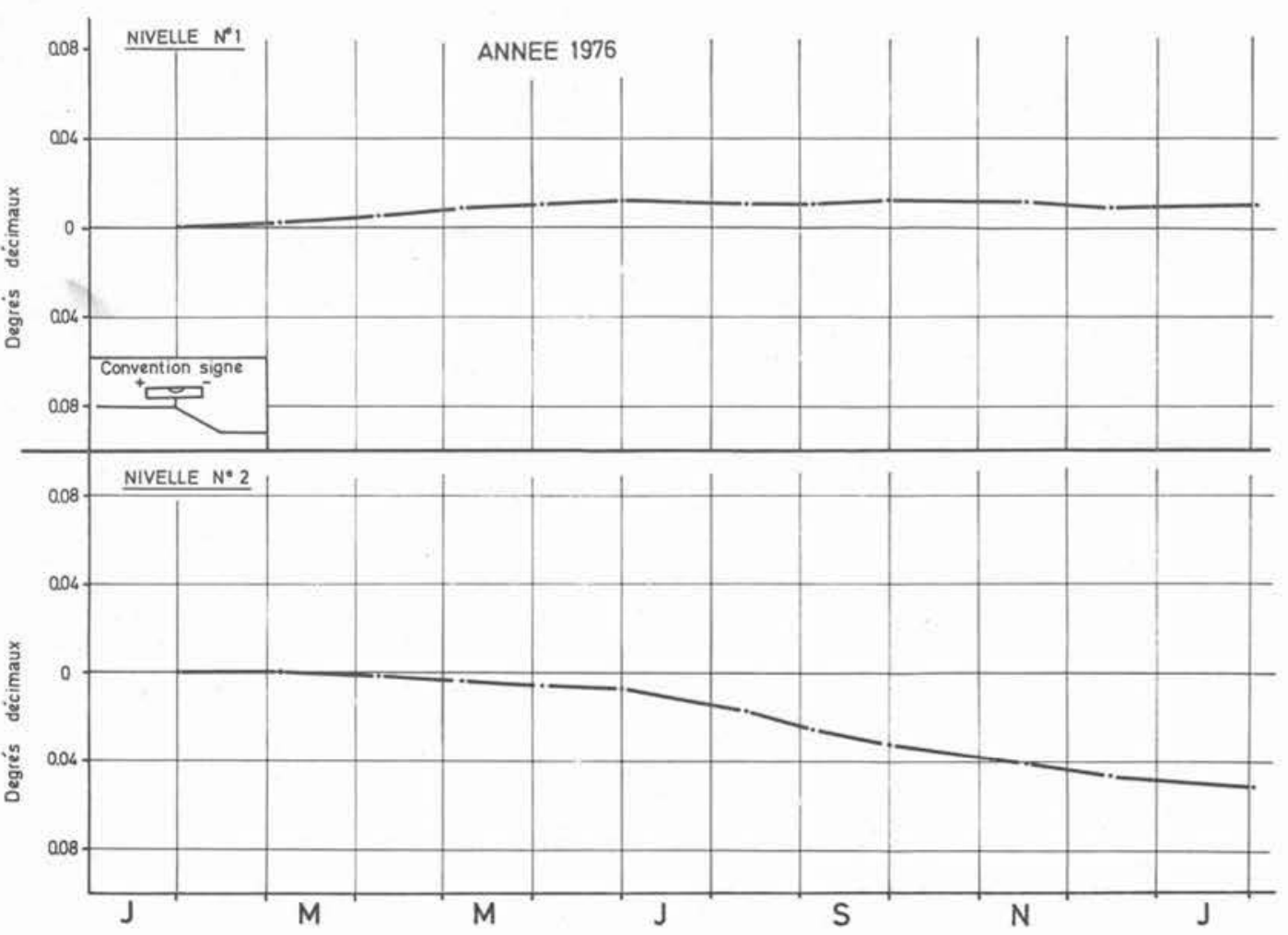

Fig. 22. - Relevé des nivelles et localisation de la rupture par inclinomètre dans un grand remblai. 
Il est impossible de tirer la cinématique du mouvement d'ensemble à partir de ces mesures. Elles indiquent localement l'existence d'un mouvement et son évolution (vitesse, accélération). La surface de la zone en mouvement peut être décrite par le suivi d'un quadrillage de ces nivelles, dont certaines sont placées en des points réputés fixes afin de servir de témoin vis-à-vis des conditions atmosphériques sur les appareils.
Les nivelles sont des appareils fiables, efficaces et peu couteux.

Placées en crête d'un remblai de $40 \mathrm{~m}$ de haut (fig. 22) dont les mouvements sont suivis d'autre part par inclinométrie, elles ont indiqué la présence de mouvements ensuite confirmés et précisés par les mesures inclinométriques dépouillées suivant la méthode décrite ci-dessus.

\section{LES DISPOSITIFS D'ALERTE}

\section{Définitions}

- Alerte : signal qui prévient d'un danger.

- Alarme : cri, appel aux armes ou frayeur subite, trouble.

(Définition du Larousse).

Les appareils de mesure dont les informations sont utilisées pour prévenir d'un danger sont donc des dispositifs d'alerte.

Tous les appareils donnant l'évolution d'une quantité (déplacement ou rotation) liée à des mouvements du terrain peuvent servir de dispositif d'alerte.

Lorsque le phénomène à surveiller se développe très rapidement, relativement aux possibilités de scrutation des mesures, seuls les dispositifs par tout ou rien peuvent convenir et donner l'alerte.

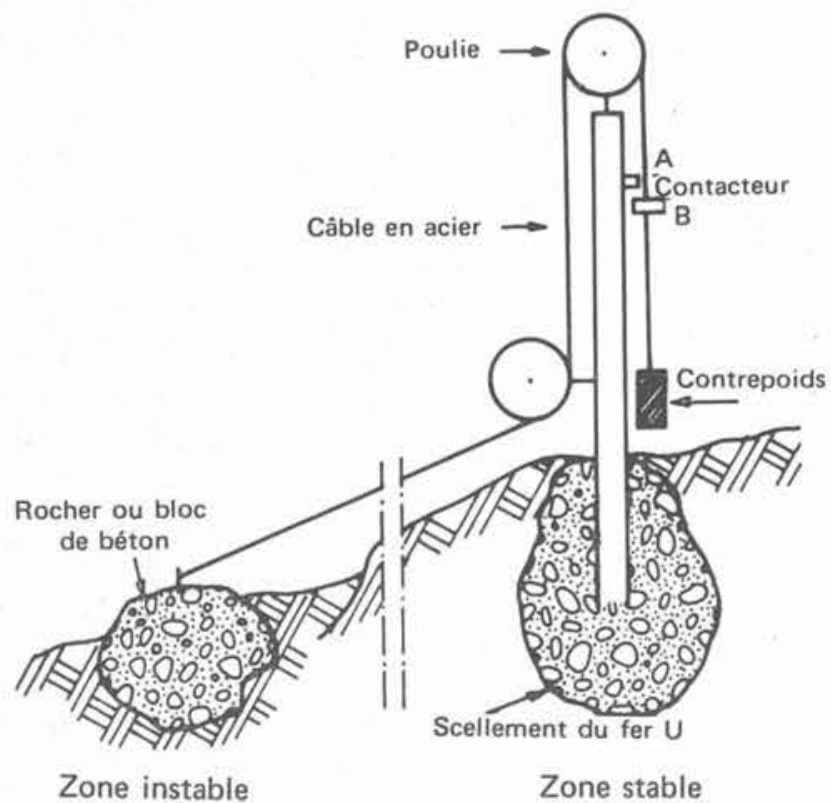

Fig. 23. - Schéma de principe du dispositif d'alerte à câble et détail du contacteur.

C'est le cas des avertisseurs à fils de rupture utilisés par la SNCF sur la ligne Culoz-Modane pour détecter la chute de blocs sur la voie, ou du dispositif à moulinet de Pontamafrey dont la mise en rotation par une coulée boueuse déclenche une trompe et des feux d'interdiction de passage sur la voie ferrée Paris-Turin.

1. Un exemple de dispositif d'alerte utilisant la mesure du déplacement de points de surface

Ce dispositif, développé par le laboratoire de Strasbourg, comprend un poteau (1) placé dans une zone stable sur lequel est monté un jeu de poulies de renvoi et un contacteur. Un câble (2) relie ce point fixe à la zone instable (fig. 23 et 24).

L'alarme qui peut être électrique ou pneumatique (corne de brume) est déclenchée par un certain déplacement du câble (définition d'un seuil de quelques millimètres à quelques centimètres).

Le poteau de contrôle est également muni d'une réglette graduée sur laquelle glisse un ou deux index liés au câble.

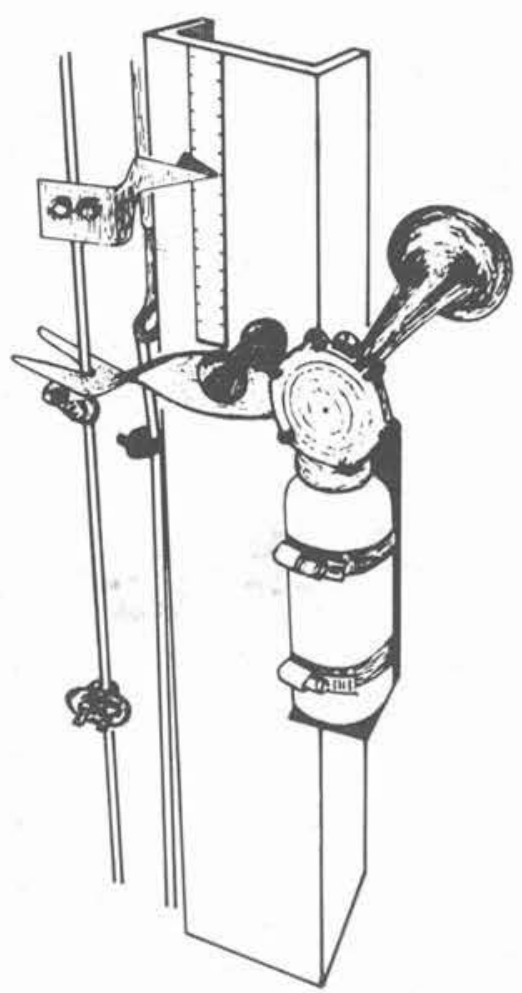

Fig. 24. - Dispositif d'alerte à câble installé sur un talus en Alsace.

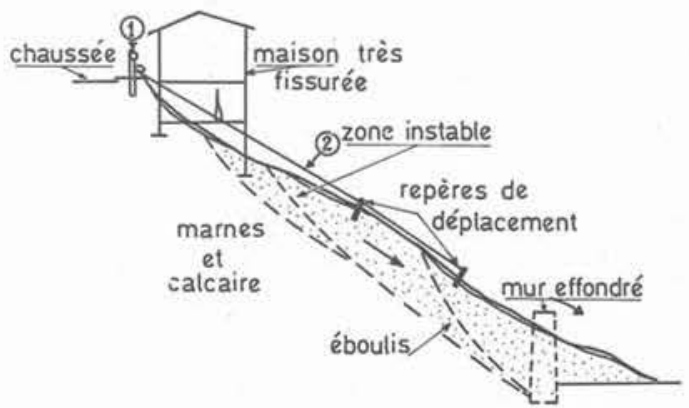




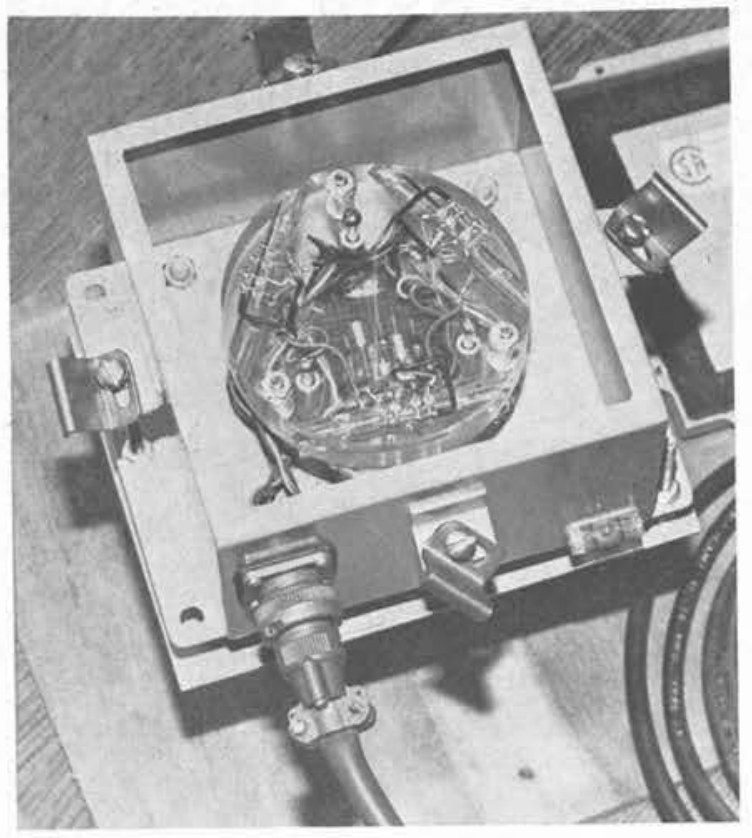

Fig. 25. - Dispositif d'alerte par niveau à bulles.

Cet appareil très rustique, à seuil réglable, a effectivement déclenché une alarme au moment du glissement d'une partie d'un talus en Alsace (seuil de déplacement $\pm 1 \mathrm{~cm}$ ).

Il permet de suivre le déplacement du point grâce à une réglette graduée. Les phénomènes de dilatation thermique et de fluage du fil peuvent cependant intervenir et rendre difficile la mesure ou provoquer la mise en route intempestive d'un signal d'alarme enlevant toute crédibilité en l'appareil. Ce point est actuellement à l'étude.

2. Un exemple de dispositif utilisant la mesure de la rotation des points de la surface du terrain

Cet appareil canadien appelé « détecteur de glissement de terrain " a été étudié à l'Ecole Polytechnique de Montréal pour le compte du Service de Géotechnique du Ministère des Richesses Naturelles du Québec (MRN) (fig. 25 et 26).

Les éléments de bases de cet appareil automatique sont trois niveaux à bulle placés à $120^{\circ}$ dans un coffret. Le déplacement de la bulle d'air est détecté électroniquement par un émetteur-récepteur infrarouge

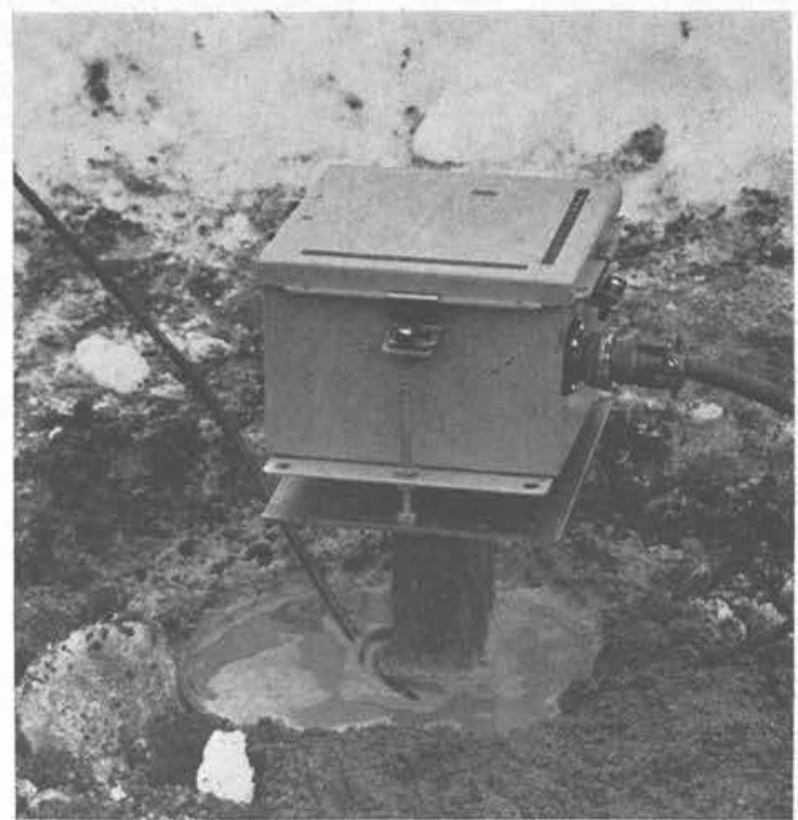

Fig. 26. - Dispositif mis en place.

dont le faisceau est plus ou moins occulté par la bulle.

Lorsqu'il y a mouvement du sol (rotation de $1.35^{\circ}$ $\pm 0.1^{\circ}$ ), la bulle d'un ou plusieurs des trois niveaux sort du faisceau infrarouge et provoque l'émission d'un signal électrique.

Les dispositifs sont reliés à des boîtes centrales qui émettent une alarme sonore lorsque deux détecteurs différents indiquent un mouvement.

De plus, chaque détecteur est muni d'une temporisation annulant les éventuelles alertes transitoires (effet de vibrations, ou de chocs sur le détecteur).

Ces appareils ont été testés longuement dans des conditions difficiles de froid, de neige et de pluie québecoises. Ce sont des dispositifs à seuil non réglable ; le choix du seuil est imposé à la construction.

Un détecteur semblable à celui-ci, utilisant des niveaux à contact de mercure ne nécessitant pas d'électronique, est étudié au laboratoire régional de Strasbourg.

\section{TRANSMISSION DE L'INFORMATION, EXPLOITATION DES MESURES}

La quantité mesurée par le capteur est le plus souvent relevée à l'endroit même du point de mesure. Si l'accès en est facile, les relevés pourront être nombreux et peu coûteux. Dans le cas contraire, ou bien on accepte un espacement dans le temps avec le risque de voir le mouvement s'accélérer et devenir dangereux entre deux mesures, ou bien on fait un effort financier de façon à augmenter le nombre de relevés.

L'importance de ce dernier point pourrait s'atténuer du fait du développement de deux techniques :

- l'utilisation de dispositifs d'alerte à faible seuil indiquant le moment où il faut faire une mesure complète (par exemple l'utilisation d'une nivelle automatique à très faible seuil à côté d'un tube inclinométrique) ;

- l'utilisation de la transmission à longue distance des mesures ou télémesure.

\section{La télémesure}

La télémesure par lignes téléphoniques est celle qui permettrait le mieux d'automatiser la lecture de capteurs placés sur un site.

Une étude de l'Ecole Polytechnique de Montréal sur ce sujet indique qu'on peut envisager :

- dans le cas de un à deux capteurs, la transmis- 
sion à l'aide d'une onde dont la fréquence varie avec l'amplitude du signal mesuré (FM) et décodée à l'aide d'un fréquencemètre ;

- dans le cas de plus de deux capteurs, l'utilisation d'un système de type " acquisition de données » transmettant les données sous forme numérique.

L'élément de sortie peut varier de l'imprimante au mini-ordinateur faisant tout le traitement des données.
La télémesure permet donc de surveiller tous tes sites où le téléphone peut accéder, de façon permanente et sans se déplacer.

Des systèmes comparables ont été développés pour la surveillance du niveau de fleuves ou de la pluviométrie mais peu, ou pas, pour la surveillance de sites instables.

\section{CONCLUSION}

La panoplie des moyens mis à la disposition de l'ingénieur pour la surveillance en déplacement des glissements est large. Les appareils de mesure tels que extensomètres, inclinomètres, capteurs de déplacement, les techniques telles que topographie et photogrammétrie peuvent lui fournir des résultats de grande qualité pour un bonne surveillance.

Quel que soit le type de surveillance adopté, son choix est lié à l'importance relative de critères tels que :

- possibilités de mise en œuvre de l'appareillage ;

- fiabilité (nécessité de doubler certains appareils);

- accessibilité du site pour les relevés;
- précision de la mesure ;

- rapidité d'exploitation et qualité de la méthode de dépouillement;

- validité des seuils des dispositifs d'alerte;

- coût.

Les dispositifs d'alerte ( « tout ou rien », à seuil réglable ou non) ne donnent aucun renseignement sur l'évolution du phénomène avant l'alerte et devraient se présenter plutôt comme des moyens complémentaires des appareils de mesures.

Quel que soit le système adopté, il faut avoir présent à l'esprit qu'une mesure ne vaut que ce que vaut le plus mauvais maillon de la chaîne qui a servi à l'obtenir.

\section{REFERENCES}

[1] BURLAND (J.B.) and MOORE (J.F.A.). - "The measurement of ground displacement around deep excavations». Field Instrumentation in Geotechnical Engineering, p. 70-84, (1973).

[2] EGGER (K.) and KELLER (W.). - New instruments, methods and their applications for geodetic deformation measurements on dams. Transactions of the Twelfth International Congress on Large Dams, Mexico (1976).

[3] HANNA (T.H.). - «Foundation Instrumentation», Trans. Tech. Publications, Clausthal (1973).

[4] LENGLET (J.). - Appareils et méthodes de surveillance des glissements de terrain. Bulletin de Liaison des Laboratoires des Ponts et
Chaussées. No spécial "Stabilité des talus ", p. 166-174 (mars 1976).

[5] PEIGNAUD (M.) et PERRIN (J.). - Inclinomètres, Bulletin de Liaison des Laboratoires des Ponts et Chaussées. Spécial T. "Remblais sur sols compressibles », p. 297-304 (mai 1973).

[6] TER STEPANIAN (G.). - In situ determination of the rheological characteristics of soils in slopes. $6^{\mathrm{e}}$ Congrès international de Mécanique des Sols et des Travaux de fondation, vol. II, p. 575-577 (1965).

[7] Photogrammétrie : applications au domaine du Génie Civil. Doc. Centre d'Actualisation Scientifique et Technique. Journées d'études INSA (avril 1974). 